\title{
Are consumption of dairy products and physical activity independently related to bone mineral density of 6-year-old children? Longitudinal and cross-sectional analyses in a birth cohort from Brazil
}

\author{
Renata M Bielemann ${ }^{1,2, *}$, Juliana dos $S V_{a z}{ }^{1,2}$, Marlos R Domingues ${ }^{3}$, \\ Alicia Matijasevich ${ }^{1,4}$, Iná S Santos ${ }^{1}$, Ulf Ekelund ${ }^{5,6}$ and Bernardo L Horta ${ }^{1}$ \\ ${ }^{1}$ Postgraduate Program in Epidemiology, Federal University of Pelotas, Rua Marechal Deodoro $1160-3^{\circ}$ andar, Pelotas - \\ RS, 96020-220, Brazil: ${ }^{2}$ School of Nutrition, Federal University of Pelotas, Pelotas, RS, Brazil: ${ }^{3}$ Postgraduate Program in \\ Physical Education, Federal University of Pelotas, Pelotas, RS, Brazil: ${ }^{4}$ Department of Preventive Medicine, School of \\ Medicine, University of São Paulo, São Paulo, SP, Brazil: ${ }^{5}$ Medical Research Council, Epidemiology Unit, University of \\ Cambridge, Cambridge, UK: ${ }^{6}$ Department of Sport Medicine, Norwegian School of Sport Sciences, Oslo, Norway
}

Submitted 24 November 2017: Final revision received 13 March 2018: Accepted 11 April 2018: First published online 16 May 2018

\begin{abstract}
Objective: To evaluate cross-sectional and longitudinal associations of consumption of dairy products and physical activity (PA) with bone mineral density (BMD). Design: Cohort study with children from the 2004 Pelotas (Brazil) Birth Cohort.

Setting: Pelotas, a medium-sized Brazilian city.

Subjects: The study started in 2004 and mothers/children were interviewed/ measured periodically from birth to age 6 years. PA was measured by maternal proxy at 4 and 6 years and by accelerometry at 6 years. Consumption of dairy products was measured using $24 \mathrm{~h}$ food recall (at 4 years) and FFQ (at 6 years). Total-body and lumbar-spine BMD $\left(\mathrm{g} / \mathrm{cm}^{2}\right)$ were measured by dual-energy X-ray absorptiometry. Results: At 6 years, BMD was measured in 3444 children and 2636 children provided data on objectively measured PA by accelerometry. Consumption of dairy products at 4 years was associated with higher lumbar-spine BMD at 6 years in boys, while current consumption was positively associated with BMD in both sexes $(P<0 \cdot 001)$. PA assessed by maternal report at 4 and 6 years of age was associated with higher BMD at 6 years in boys. PA assessed by accelerometry was positively related to total-body and lumbar-spine BMD in boys and lumbar-spine BMD in girls. We did not find evidence for an interaction between PA and consumption of dairy products on BMD.

Conclusions: We observed positive and independent longitudinal and crosssectional associations between consumption of dairy products and PA with BMD in the total body and at the lumbar spine in young children.
\end{abstract}

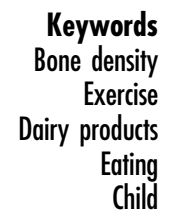

The physical capability across the lifespan is influenced by early factors that are related to the amount of 'biological capital' acquired during growing years. The peak of physical capability, as part of the 'biological capital', allows individuals to remain above a critical threshold of risk for adverse outcomes later in life ${ }^{(1)}$. Peak bone mass is one such example of the physical capability, partly influenced by early-life factors, contributing to reach the 'full genetic potential' which may then prevent osteoporosis-related fractures in the future ${ }^{(2)}$. The amount of nutrients available and the time spent in physical activity (PA), especially weight-bearing activities, are important in this context because the skeleton may not achieve its 'full genetic potential' if the supply of nutrients and/or mechanical loading during childhood and youth is insufficient ${ }^{(2)}$.

The importance of diet during childhood and adolescence to promote peak bone mass is well established and based on the role of dietary $\mathrm{Ca}^{(2)}$. Dairy products are probably the best dietary sources of $\mathrm{Ca}$ in childhood and dietary $\mathrm{Ca}$ is more strongly associated with bone mass 
compared with similar amounts provided by supplementation $^{(3)}$. Thus, dairy products are considered an efficient source of nutrients for the bone because, besides $\mathrm{Ca}$, they contain protein, $\mathrm{Mg}, \mathrm{K}, \mathrm{Zn}$ and $\mathrm{P}$ that are important nutrients in the construction of bone tissue ${ }^{(4)}$.

PA is an important modifiable factor influencing the development of bone mass. Previous randomized controlled trials reported greater bone mineral density (BMD) in children allocated to PA compared with controls ${ }^{(5,6)}$, confirmed by observational research ${ }^{(7)}$.

Although the positive relationship between dairy products, PA and BMD is recognized, some gaps remain. For example, it is unclear if the effects of $\mathrm{Ca}$ intake and PA on BMD are additive and/or interact. In addition, it is unclear if the response to PA and $\mathrm{Ca}$ intake on BMD is different among boys and girls before puberty ${ }^{(5)}$. Finally, there is no consensus on whether the positive effect of PA and Ca intake on BMD is cumulative during childhood, depending on how long children are exposed to higher levels of PA or Ca intake. Prospective examinations of the effect of PA and dietary factors, such as consumption of dairy products, on BMD among free-living young children are also scarce.

Thus, the aims of the present study were to: (i) assess the cross-sectional and longitudinal associations of consumption of dairy products and PA with BMD; (ii) examine the dose-response association of each individual exposure with the outcome; and (iii) evaluate if associations between exposures and BMD are additive or multiplicative. We used data from 4- and 6-year-old children belonging to the 2004 Pelotas (Brazil) Birth Cohort.

\section{Methods}

Pelotas is a medium-sized city in Rio Grande do Sul (southern Brazil) with more than 300000 inhabitants. In 2004, all maternity hospitals were visited daily from 1 January to 31 December and mothers were invited to participate, interviewed and their newborns were examined. All children and their mothers have been followed-up since then.

Follow-up assessments of the 2004 Pelotas Birth Cohort study were conducted during home visits at mean ages of 3.0 (SD 0.1), 11.9 (SD 0.2), 23.9 (SD 0.4) and 49.5 (SD 1.7) months and at the research clinic at 6.8 (SD 0.3 ) years. Detailed methods of the cohort are available elsewhere ${ }^{(8)}$.

Total-body and lumbar-spine (L1-L4) BMD $\left(\mathrm{g} / \mathrm{cm}^{2}\right)$ were measured by dual-energy X-ray absorptiometry (DXA; Lunar Prodigy Advance ${ }^{\mathrm{TM}}$, GE Healthcare, Germany). Data were not collected from disabled children or those presenting metal surgical implants and irremovable metal items. In total, 3444 children were scanned by DXA during the visit to the research clinic at mean age $6 \cdot 8$ years.

Interviews included a questionnaire to assess food consumption (at 4 and 6 years); a $24 \mathrm{~h}$ food recall (at
4 years) including milk, formula and yoghurt consumption; and an FFQ based on the 12 months prior to the interview at 6 years including whole and skimmed milk, yoghurt and cheese. Also, changes in consumption patterns from 4 to 6 years were evaluated based on the recommended daily ingestion of at least three portions of milk or dairy products according to the previous Brazilian Dietary Guidelines ${ }^{(9)}$.

PA was assessed by maternal report when children were 4 years old using the last question of the Netherlands Physical Activity Questionnaire (NPAQ) ${ }^{(10)}$. Mothers were asked to classify their children as 'about equal', 'always' or 'almost always' less or more physically active compared with other children of the same age. Scores ranged from 1 to 5 points from less to more active option. This question from NPAQ showed a correlation coefficient of $0 \cdot 27$ with daily minutes of moderate-to-vigorous PA (MVPA) ${ }^{(10)}$. Children were categorized into three PA groups according to maternal perception: 'above average' ( 4 or 5 points), 'average' (3 points) and 'below average' ( 1 or 2 points). We also considered the PA change from 4 to 6 years based on maternal perception using 'above average' as the category of reference.

PA was objectively measured by accelerometry in a subsample of children at 6.8 years ( $n$ 2636, 69\% of eligible children). The GENEActiv accelerometer is waterproof and measures acceleration in three axes $(x, y, z)$ within $\mathbf{a} \pm 8 \boldsymbol{g}$ dynamic range with a sampling frequency set at $85.7 \mathrm{~Hz}$. Data are stored directly as sampled from the microelectromechanical systems chip (unfiltered) and expressed in units of $\mathrm{m} \boldsymbol{g}\left(1000 \mathrm{~m} \boldsymbol{g}=1 \boldsymbol{g}=9 \cdot 81 \mathrm{~m} / \mathrm{s}^{2}\right)$. The accelerometer was placed at the children's non-dominant wrist and PA was assessed using a $24 \mathrm{~h}$ protocol for four to seven freeliving days including at least one weekend day in all participants. Participants who visited the clinic on a Monday, Tuesday or Wednesday were monitored until the following Monday, whereas those who visited the clinic on a Thursday, Friday or Saturday were monitored until the following Wednesday. Following the free-living measurements, accelerometers were collected by the research team at the participants' home. Children who were disabled or living in other cities were excluded from the measurements. More details on the collection of data from accelerometry are given in a previous publication ${ }^{(11)}$.

Accelerometry data were analysed with the GENEActiv software. Binary data were analysed with the $\mathrm{R}$ package GGIR (http://www.cran.r-project.org/web/packages/ GGIR/vignettes/GGIR.html\#citing-ggir). Detailed signal processing included verification of sensor calibration error using local gravity as reference, detection of sustained abnormally high values, non-wear detection, and exclusion of the first $10 \mathrm{~h}$ and last $20 \mathrm{~h}$ of the measurement. Calculation of the vector magnitude of activity-related acceleration was made using the Euclidian Norm minus $1 \boldsymbol{g}$ (ENMO: $\sqrt{x^{2}+y^{2}+z^{2}}-1 \mathbf{g}$ ) with any negative values rounded up to zero. Data were imputed for periods with 
invalid data and the average of similar time points on different days of the measurement was used. Valid data were present for every $15 \mathrm{~min}$ period in a $24 \mathrm{~h}$ cycle (even when scattered over multiple days). Procedures in the accelerometry analyses were conducted according to previous publications ${ }^{(12-14)}$.

Summary measurements were the average magnitude of wrist acceleration (overall volume, ENMO, mg) and estimated time spent in $10 \mathrm{~min}$ bouts of MVPA. Daily time spent in MVPA was based on an intensity threshold of $100 \mathrm{mg}$ (for each $5 \mathrm{~s}$ epoch data and $10 \mathrm{~min}$ bouts). Sensitivity analyses were also performed using time spent above a $200 \mathrm{mg}$ cut-off point. Data from Hildebrand et al. suggest that $100 \mathrm{mg}$ is similar to walking at $3 \mathrm{~km} / \mathrm{h}$ in children and approximately $200 \mathrm{~m} \boldsymbol{g}$ is equal to MVPA ${ }^{(15)}$. Data from accelerometry (acceleration in $\mathbf{m} \boldsymbol{g}$ and MVPA in $\mathrm{min} / \mathrm{d}$ ) were analysed as continuous variables and categorized into quartiles.

The following variables were considered as potential confounders: child's skin colour (white, black, brown or other); family income at birth (asked in the perinatal interview, being the sum of the earnings of the household members); maternal schooling at birth (in complete years of schooling); birth weight (measured by the hospital staff using paediatric scales (Filizola, São Paulo, Brazil) accurate to $10 \mathrm{~g}$ and calibrated weekly with standard weights; in grams); maternal smoking during pregnancy (asked in the perinatal interview; yes/no); maternal age at birth; breastfeeding duration (from information asked in all follow-up interviews up to 4 years of age; in months); and current height (measured to the nearest $1 \mathrm{~mm}$, using a wooden stadiometer, in standing position).

All statistical analyses were performed with the statistical software package Stata version 12 and stratified by sex. Absolute and relative frequency of main exposures and confounders were described, as well as mean and standard deviation of both outcomes. Unadjusted and adjusted analyses were performed using linear regression and $P$ values were obtained by Wald's test for heterogeneity, using PA variables by proxy report and consumption of dairy products at both ages as ordinal variables ( $\beta$ coefficients calculated represent the difference in BMD for each category in relation to the reference group). Adjusted analyses included all confounders listed previously. Analyses did not include body weight as a confounder since it was not related to total-body or lumbar-spine BMD. Statistical procedures were performed using PA variables by proxy report and consumption of dairy products at both ages as ordinal variables and by classification according to status in exposures at both ages. To examine potential effect modification (in the present study, when the $P$ values for the interaction term inserted in the analyses were $<0.05$ ), we included interaction terms (exposure $\times$ sex) for both exposures and also for the association of PA with BMD according to consumption of dairy products at each age $(\mathrm{PA} \times$ consumption of dairy products at each age). The significance level was set at $5 \%$.

All follow-ups of the Pelotas 2004 Birth Cohort Study were approved by the Ethics Committee of the Federal University of Pelotas Medical School. All mothers signed an informed consent before any data collection.

\section{Results}

In 2004, 4231 children were enrolled in the cohort. Between birth and follow-up at age 6 years, ninety-five children died. Of the original cohort, 3722 children were located and interviewed (follow-up rate $=90 \cdot 2 \%$ ). Of these, 3444 (92.5\%) children had valid data from the DXA scans whereas 2636 children provided at least two valid days of PA assessed by accelerometry (69.1\%). Children without data on objectively measured PA had higher total-body BMD than children with valid data (see online supplementary material, Supplemental Table 1). There was no other statistical difference between groups.

Table 1 shows the characteristics of boys and girls. Most mothers had at least 8 years of schooling and about $25 \%$ were black or brown. More than $80 \%$ and about $30 \%$ of children reported consumption of cow's milk and yoghurt at least once during the $24 \mathrm{~h}$ prior to the interview at 4 years of age, respectively. Approximately $30 \%$ of boys and $20 \%$ of girls reported cow's milk consumption at least three times daily at 6 years of age, whereas most of the children (53\%) consumed dairy products, except for milk, at least once daily at age 6 years. Mothers classified approximately $50 \%$ and $40 \%$ of their children as 'above average' for PA at 4 and 6 years of age, respectively. More boys than girls were categorized into the two highest quartiles according to objectively measured PA. Totalbody BMD at 6 years of age was on average greater in boys whereas lumbar-spine BMD was greater in girls.

The association between consumption of dairy products, mothers' perception of children's PA and total-body BMD is displayed in Table 2. Higher consumption of dairy products at both ages was positively associated with higher total-body BMD at 6 years in boys and girls $(P<$ $0 \cdot 001)$. The magnitude of the association was greater in boys for consumption of dairy products at 6 years than in girls. Consuming dairy products at least once daily was positively associated with total-body BMD; however, the magnitude of the association was greater in those consuming dairy products three or more times daily. Boys classified by their mothers as 'below average' for PA at 4 and 6 years presented total-body BMD 0.014 (95\% CI $-0.024,-0.005) \mathrm{g} / \mathrm{cm}^{2}$ and 0.017 (95\% CI -0.024, -0.009) $\mathrm{g} / \mathrm{cm}^{2}$ lower, respectively, compared with those classified as 'above average' for PA. Girls classified by their mothers as 'below average' for PA at 6 years showed 0.009 (95\% CI $-0 \cdot 017,-0 \cdot 001) \mathrm{g} / \mathrm{cm}^{2}$ lower total-body BMD than those classified as 'above average' for PA. 
Table 1 Sociodemographic characteristics, consumption of dairy products, physical activity (PA) and bone mineral density (BMD) in children belonging to the 2004 Pelotas (Brazil) Birth Cohort

\begin{tabular}{|c|c|c|c|c|}
\hline & \multicolumn{2}{|c|}{ Boys } & \multicolumn{2}{|c|}{ Girls } \\
\hline & $n$ or Mean & $\%$ or SD & $n$ or Mean & $\%$ or SD \\
\hline \multicolumn{5}{|l|}{ Maternal schooling (years) } \\
\hline $0-4$ years & 264 & $15 \cdot 0$ & 253 & 15.4 \\
\hline $5-8$ years & 734 & 41.6 & 701 & $42 \cdot 6$ \\
\hline $9-11$ years & 585 & 33.1 & 553 & $33 \cdot 6$ \\
\hline$\geq 12$ years & 182 & $10 \cdot 3$ & 140 & 8.5 \\
\hline \multicolumn{5}{|l|}{ Skin colour } \\
\hline White & 1176 & $67 \cdot 3$ & 1118 & $67 \cdot 7$ \\
\hline Black & 233 & $13 \cdot 3$ & 192 & 11.6 \\
\hline Brown & 256 & 14.7 & 240 & 14.5 \\
\hline Other & 82 & 4.7 & 102 & $6 \cdot 2$ \\
\hline \multicolumn{5}{|c|}{ Daily frequency of consumption of cow's milk at 4 years } \\
\hline$<1$ & 311 & $18 \cdot 0$ & 295 & $18 \cdot 2$ \\
\hline $1-2$ & 680 & $39 \cdot 1$ & 681 & $42 \cdot 1$ \\
\hline 3 & 547 & 31.6 & 484 & 29.9 \\
\hline$\geq 4$ & 195 & 11.3 & 157 & $9 \cdot 7$ \\
\hline \multicolumn{5}{|c|}{ Daily frequency of consumption of yoghurt at 4 years } \\
\hline$<1 \quad<-1<0$ & 1112 & $64 \cdot 1$ & 1086 & $67 \cdot 1$ \\
\hline 1 & 417 & $24 \cdot 1$ & 362 & 22.4 \\
\hline$\geq 2$ & 204 & $11 \cdot 8$ & 169 & $10 \cdot 5$ \\
\hline \multicolumn{5}{|c|}{ Daily frequency of consumption of cow's milk at 6 years } \\
\hline$<1$ & 424 & $26 \cdot 0$ & 443 & 28.7 \\
\hline $1-2$ & 715 & 43.7 & 777 & $50 \cdot 2$ \\
\hline 3 & 447 & 27.4 & 293 & 18.9 \\
\hline$\geq 4$ & 48 & 2.9 & 34 & $2 \cdot 2$ \\
\hline \multicolumn{5}{|c|}{ Daily frequency of consumption of yoghurt and cheese at 6 years } \\
\hline$<1$ & 742 & 45.5 & 725 & $47 \cdot 0$ \\
\hline 1 & 447 & 27.5 & 445 & 28.9 \\
\hline$\geq 2$ & 439 & $27 \cdot 0$ & 372 & $24 \cdot 1$ \\
\hline \multicolumn{5}{|l|}{ Maternal perception of PA at 4 years } \\
\hline Below average & 94 & 5.4 & 68 & 4.2 \\
\hline Average & 800 & $46 \cdot 2$ & 716 & $44 \cdot 3$ \\
\hline Above average & 837 & 48.4 & 832 & 51.5 \\
\hline \multicolumn{5}{|l|}{ Maternal perception of PA at 6 years } \\
\hline Below average & 188 & $10 \cdot 8$ & 168 & $10 \cdot 2$ \\
\hline Average & 845 & 48.4 & 808 & $49 \cdot 1$ \\
\hline Above average & 712 & $40 \cdot 8$ & 671 & $40 \cdot 7$ \\
\hline Overall PA by accelerometer at 6 years $(\mathrm{mg})$ & 64.0 & 17.4 & 54.8 & 13.5 \\
\hline MVPA by accelerometer at 6 years $(\min )$ & $55 \cdot 6$ & 38.4 & 33.4 & $186 \cdot 3$ \\
\hline Total-body BMD $\left(\mathrm{g} / \mathrm{cm}^{2}\right)$ & 0.834 & 0.048 & 0.819 & 0.049 \\
\hline Lumbar-spine (L1-L4) BMD (g/cm²) & 0.646 & 0.079 & 0.667 & 0.088 \\
\hline
\end{tabular}

MVPA, moderate-to-vigorous PA.

The consumption of dairy products three or more times daily at 4 years of age was positively associated with higher lumbar-spine BMD at 6 years in boys (Table 3 ). The daily frequency of consumption of dairy products at 6 years was positively associated with lumbar-spine BMD in both sexes. Consuming dairy products at least once daily at age 6 years was positively associated with lumbar-spine BMD in boys, whereas in girls the positive association was observed only in those who consumed dairy products three times daily. Boys classified as 'below average' for PA had on average $0.029(95 \% \mathrm{CI}-0.045,-0.014) \mathrm{g} / \mathrm{cm}^{2}$ and 0.025 $(95 \% \mathrm{CI}-0.037,-0.013) \mathrm{g} / \mathrm{cm}^{2}$ lower lumbar-spine BMD at 6 years than boys classified as 'above average' for PA at 4 and 6 years of age, respectively. Boys classified as 'average' for PA at both ages also had lower lumbar-spine BMD compared with more active boys. No association was found between maternal perception of PA and lumbar-spine BMD in girls at age 6 years.
The association between objectively measured PA and BMD is shown in Table 4. Both overall PA $(P=0.018$ for total-body BMD; $P=0.002$ for lumbar-spine BMD) and time spent in MVPA ( $P=0.009$ for total-body BMD; $P=$ 0.024 for lumbar-spine BMD) were positively associated with total-body and lumbar-spine BMD in boys. In girls, overall PA and time spent in MVPA were positively associated with lumbar-spine $\operatorname{BMD}(P=0.002$ and $P=$ $0 \cdot 029$, respectively). Results using information on objectively measured PA with the $200 \mathrm{mg}$ cut-off point were not statistically associated with BMD (see online supplementary material, Supplemental Table 2).

Figure 1 shows that achieving the recommended levels of dairy product intake ( $\geq 3$ portions of milk or dairy products daily) at both 4 and 6 years of age was positively associated with total-body BMD at 6 years of age in boys $(P<0.001)$. Girls who adhered to the recommendation for consumption of dairy products at age 6 years or at both 


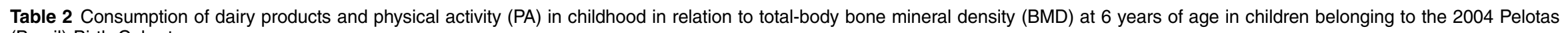
(Brazil) Birth Cohort

\begin{tabular}{|c|c|c|c|c|c|c|c|c|c|c|}
\hline & \multicolumn{10}{|c|}{ Total-body BMD $\left(\mathrm{g} / \mathrm{cm}^{2}\right)$} \\
\hline & \multicolumn{5}{|c|}{ Boys } & \multicolumn{5}{|c|}{ Girls } \\
\hline & \multirow[b]{2}{*}{$n$} & \multicolumn{2}{|c|}{ Crude } & \multicolumn{2}{|c|}{ Adjusted } & \multirow[b]{2}{*}{$n$} & \multicolumn{2}{|c|}{ Crude } & \multicolumn{2}{|c|}{ Adjusted } \\
\hline & & $\beta$ coefficient & $95 \% \mathrm{Cl}$ & $\beta$ coefficient & $95 \% \mathrm{Cl}$ & & $\beta$ coefficient & $95 \% \mathrm{Cl}$ & $\beta$ coefficient & $95 \% \mathrm{Cl}$ \\
\hline Daily frequency of dairy products at 4 years* & & \multirow{2}{*}{\multicolumn{2}{|c|}{$P<0.001$}} & \multicolumn{2}{|c|}{$P<0.001$} & & \multicolumn{2}{|c|}{$P<0.001$} & \multicolumn{2}{|c|}{$P<0.001$} \\
\hline$<1$ & 176 & Ref. & & \multicolumn{2}{|c|}{ Ref. } & 163 & \multicolumn{2}{|c|}{ Ref. } & \multicolumn{2}{|c|}{ Ref. } \\
\hline $1-2$ & 583 & 0.019 & $0.011,0.027$ & 0.011 & $0.004,0.019$ & 614 & 0.025 & $0.016,0.033$ & 0.018 & $0.009,0.026$ \\
\hline 3 & 529 & 0.030 & $0.022,0.038$ & 0.021 & $0.013,0.028$ & 489 & 0.030 & $0.022,0.039$ & 0.022 & $0.014,0.030$ \\
\hline$\geq 4$ & 445 & 0.031 & $0.023,0.039$ & 0.022 & $0.014,0.030$ & 351 & 0.033 & $0.025,0.042$ & 0.025 & $0.016,0.034$ \\
\hline Daily frequency of dairy products at 6 years* & & \multicolumn{2}{|c|}{$P<0.001$} & \multicolumn{2}{|c|}{$P<0.001$} & & \multicolumn{2}{|c|}{$P<0.001$} & \multicolumn{2}{|c|}{$P<0.001$} \\
\hline$<1$ & 223 & \multicolumn{2}{|c|}{ Ref. } & \multicolumn{2}{|c|}{ Ref. } & 257 & \multicolumn{2}{|c|}{ Ref. } & \multicolumn{2}{|c|}{ Ref. } \\
\hline $1-2$ & 621 & 0.021 & $0.014,0.029$ & 0.014 & $0.007,0.021$ & 666 & 0.016 & $0.009,0.023$ & 0.007 & $0.001,0.014$ \\
\hline 3 & 359 & 0.034 & $0.026,0.042$ & 0.024 & $0.016,0.032$ & 321 & 0.027 & $0.019,0.035$ & 0.017 & $0.010,0.025$ \\
\hline$\geq 4$ & 424 & 0.036 & $0.028,0.043$ & 0.025 & $0.018,0.033$ & 297 & 0.026 & $0.018,0.034$ & 0.019 & $0.012,0.027$ \\
\hline Maternal perception of PA at 4 years $\dagger$ & & \multicolumn{2}{|c|}{$P=0.020$} & \multicolumn{2}{|c|}{$P=0.009$} & & \multicolumn{2}{|c|}{$P=0.045$} & \multicolumn{2}{|c|}{$P=0.110$} \\
\hline Below average & 94 & -0.014 & $-0.025,-0.004$ & -0.014 & $-0.024,-0.005$ & 68 & -0.015 & $-0.027,-0.003$ & -0.011 & $-0.023,0.000$ \\
\hline Average & 800 & -0.001 & $-0.006,0.003$ & -0.004 & $-0.008,0.001$ & 716 & 0.000 & $-0.005,0.005$ & -0.003 & $-0.008,0.002$ \\
\hline Above average & 837 & & Ref. & & Ref. & 832 & & Ref. & & Ref. \\
\hline Maternal perception of PA at 6 years $\dagger$ & & & 0.024 & & 0.001 & & & 0.144 & & 0.060 \\
\hline Below average & 188 & -0.011 & $-0.018,-0.003$ & -0.017 & $-0.024,-0.009$ & 168 & -0.005 & $-0.014,0.003$ & -0.009 & $-0.017,-0.001$ \\
\hline Average & 845 & -0.002 & $-0.007,0.002$ & -0.006 & $-0.011,-0.002$ & 808 & 0.002 & $-0.002,0.007$ & 0.000 & $-0.005,0.005$ \\
\hline Above average & 712 & & Ref. & & Ref. & 671 & & Ref. & & Ref. \\
\hline
\end{tabular}

Ref., reference category.

Adjusted for skin colour, family income at birth, maternal schooling, birth weight, maternal smoking during the pregnancy, maternal age at birth, breast-feeding duration and current height.

†Adjusted for current consumption of dairy products. 
Table 3 Consumption of dairy products and physical activity (PA) in childhood in relation to lumbar-spine (L1-L4) bone mineral density (BMD) at 6 years of age in children belonging to the 2004 Pelotas (Brazil) Birth Cohort

\begin{tabular}{|c|c|c|c|c|c|c|c|c|c|c|}
\hline & \multicolumn{10}{|c|}{ Lumbar-spine BMD (g/cm²) } \\
\hline & \multicolumn{5}{|c|}{ Boys } & \multicolumn{5}{|c|}{ Girls } \\
\hline & \multirow[b]{2}{*}{$n$} & \multicolumn{2}{|c|}{ Crude } & \multicolumn{2}{|c|}{ Adjusted } & \multirow[b]{2}{*}{$n$} & \multicolumn{2}{|c|}{ Crude } & \multicolumn{2}{|c|}{ Adjusted } \\
\hline & & $\beta$ coefficient & $95 \% \mathrm{Cl}$ & $\beta$ coefficient & $95 \% \mathrm{Cl}$ & & $\beta$ coefficient & $95 \% \mathrm{Cl}$ & $\beta$ coefficient & $95 \% \mathrm{Cl}$ \\
\hline Daily frequency of dairy products at 4 years ${ }^{*}$ & & \multicolumn{2}{|c|}{$P<0.001$} & \multicolumn{2}{|c|}{$P<0.001$} & & \multicolumn{2}{|c|}{$P=0.003$} & \multicolumn{2}{|c|}{$P=0.398$} \\
\hline$<1$ & 176 & \multicolumn{2}{|c|}{ Ref. } & \multicolumn{2}{|c|}{ Ref. } & 160 & \multicolumn{2}{|c|}{ Ref. } & \multicolumn{2}{|c|}{ Ref. } \\
\hline $1-2$ & 577 & 0.019 & $0.005,0.032$ & 0.006 & $-0.006,0.019$ & 606 & 0.028 & $0.012,0.044$ & 0.012 & $-0.002,0.027$ \\
\hline 3 & 525 & 0.029 & $0.015,0.042$ & 0.014 & $0.001,0.026$ & 480 & 0.026 & $0.010,0.042$ & 0.011 & $-0.004,0.026$ \\
\hline$\geq 4$ & 445 & 0.036 & $0.022,0.051$ & 0.023 & $0.010,0.036$ & 347 & 0.029 & $0.012,0.046$ & 0.012 & $-0.003,0.028$ \\
\hline Daily frequency of dairy products at 6 years* & & \multicolumn{2}{|c|}{$P<0.001$} & \multicolumn{2}{|c|}{$P=0.009$} & & \multicolumn{2}{|c|}{$P<0.001$} & \multicolumn{2}{|c|}{$P=0.031$} \\
\hline$<1$ & 222 & \multicolumn{2}{|c|}{ Ref. } & \multicolumn{2}{|c|}{ Ref. } & 252 & \multicolumn{2}{|c|}{ Ref. } & \multicolumn{2}{|c|}{ Ref. } \\
\hline $1-2$ & 616 & 0.026 & $0.014,0.038$ & 0.012 & $0.001,0.023$ & 655 & 0.024 & $0.011,0.037$ & 0.010 & $-0.002,0.022$ \\
\hline 3 & 358 & 0.040 & $0.027,0.053$ & 0.021 & $0.009,0.033$ & 318 & 0.033 & $0.019,0.045$ & 0.021 & $0.007,0.035$ \\
\hline$\geq 4$ & 418 & 0.035 & $0.023,0.048$ & 0.016 & $0.004,0.028$ & 292 & 0.022 & $0.007,0.037$ & 0.013 & $-0.001,0.027$ \\
\hline Maternal perception of PA at 4 years $\dagger$ & & \multicolumn{2}{|c|}{$P<0.001$} & \multicolumn{2}{|c|}{$P<0.001$} & & \multicolumn{2}{|c|}{$P=0.120$} & \multicolumn{2}{|c|}{$P=0.167$} \\
\hline Below average & 94 & -0.033 & $-0.050,-0.016$ & -0.029 & $-0.045,-0.014$ & 66 & -0.024 & $-0.046,-0.001$ & -0.020 & $-0.041,0.001$ \\
\hline Average & 795 & -0.007 & $-0.015,0.001$ & -0.010 & $-0.018,-0.003$ & 706 & -0.001 & $-0.010,0.008$ & -0.003 & $-0.011,0.006$ \\
\hline Above average & 832 & & Ref. & & Ref. & 820 & & ef. & & Ref. \\
\hline Maternal perception of PA at 6 years $\dagger$ & & & 0.019 & & 0.001 & & & 0.419 & $P=$ & 0.163 \\
\hline Below average & 188 & -0.018 & $-0.031,-0.005$ & -0.025 & $-0.037,-0.013$ & 169 & -0.009 & $-0.025,0.006$ & -0.013 & $-0.027,0.001$ \\
\hline Average & 838 & -0.006 & $-0.014,0.002$ & -0.011 & $-0.018,-0.003$ & 795 & 0.000 & $-0.009,0.010$ & 0.000 & $-0.009,0.008$ \\
\hline Above average & 707 & & Ref. & & Ref. & 658 & & ef. & & Ref. \\
\hline
\end{tabular}

Ref., reference category.

Adjusted for skin colour, family income at birth, maternal schooling, birth weight, maternal smoking during the pregnancy, maternal age at birth, breast-feeding duration and current height.

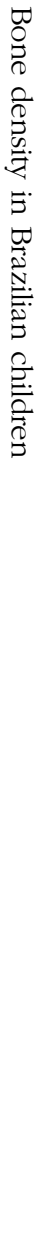

†Adjusted for current consumption of dairy products. 
time points had greater total-body BMD at age 6 years than girls who never adhered to the recommendation $(P<0 \cdot 001)$. For lumbar-spine BMD at 6 years, boys who reached the recommended consumption of dairy products at 4 years or at both time points had greater BMD than boys who never adhered to the recommendation $(P<0 \cdot 001)$. In girls, the positive association was observed only when there was adequate consumption at 6 years $(\beta=0.016$; $95 \%$ CI 0.002 , 0.030).

Boys classified by their mothers as 'above average' for PA only at 6 years or at both ages had greater total-body BMD at 6 years than boys never classified as 'above average' $(P=0 \cdot 002)$. For lumbar-spine BMD, boys classified as 'above average' for PA at any age had greater BMD than boys never classified as 'above average', although a higher $\beta$ coefficient was found in always above average boys $(P<0 \cdot 001)$. Variation on PA (maternal perception) was not related to either total-body or lumbar-spine BMD in girls ( $P=0.472$ and $P=0.578$, respectively).

Results presented are stratified by sex, although tests for effect modification of each exposure on outcomes according to sex showed no statistical significance for most exposures except for variation in adequate consumption of dairy products from 4 to 6 years $(P=0.021)$ with lumbar-spine BMD.

When the relationship between PA and BMD was stratified according to adequacy of consumption of dairy products, we observed a positive association of being classified as 'above average' for PA at 4 years on BMD with consumption of dairy products lower than three times daily in girls (total-body BMD, $\beta=0.021 ; 95 \%$ CI 0.006 , 0.036; lumbar-spine BMD, $\beta=0 \cdot 039$; $95 \%$ CI 0.014, 0.064). On the other hand, the same analysis indicated that boys who were 'average' or 'above average' for PA at 4 and 6 years had greater lumbar-spine BMD only if consumption of dairy products was adequate (PA at 4 years: 'average', $\beta=0.029$; $95 \%$ CI 0.006, 0.052; 'above average', $\beta=0.042 ; 95 \%$ CI 0.020, 0.065; PA at 6 years: 'average', $\beta=0.025 ; 95 \%$ CI 0.008, 0.041; 'above average', $\beta=0.036$; $95 \%$ CI 0.019, 0.053). However, when using objectively measured PA, a positive association was observed between PA and lumbar-spine BMD in those boys who reported an inadequate consumption of dairy products $(\beta=0.0006$; $95 \%$ CI 0.0002, 0.0009). This was not observed among girls.

\section{Discussion}

The present study assessed the cross-sectional and longitudinal associations between PA and consumption of dairy products and BMD among children from a Southern Brazilian birth cohort. To our knowledge, it is the first observational prospective study to report these associations in children from Latin America. The consumption of dairy products and PA seem to be equally important to BMD in childhood. Associations of greater magnitude were found for total-body 
(a)

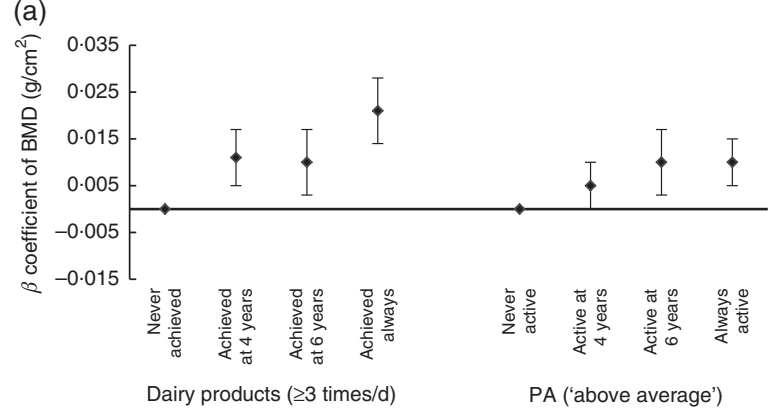

(c)

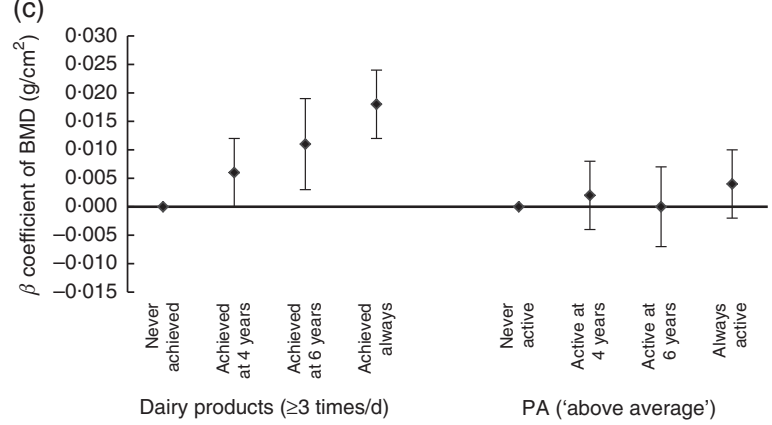

(b)

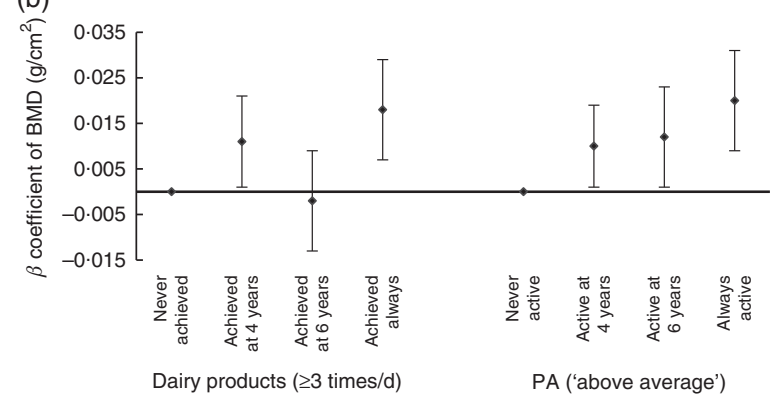

(1)

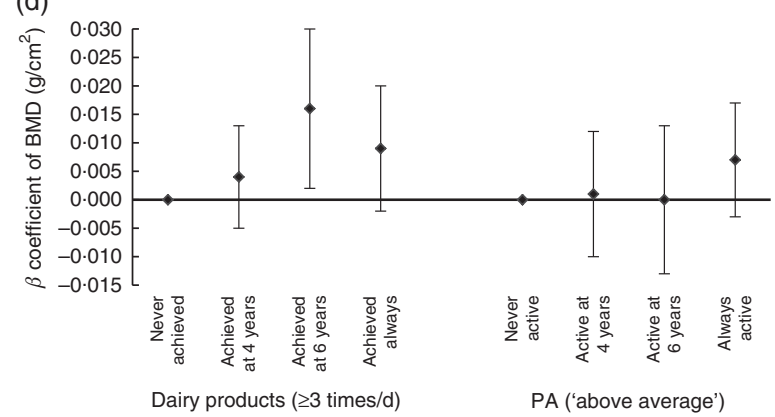

Fig. 1 Association of variation in adequate consumption of dairy products and physical activity (PA) during childhood with bone mineral density (BMD) of children from the 2004 Pelotas (Brazil) Birth Cohort at 6 years of age: (a) total-body BMD in boys; (b) lumbar-spine (L1-L4) BMD in boys; (c) total-body BMD in girls; (d) lumbar-spine (L1-L4) BMD in girls. Values are $\beta$ coefficients with their $95 \% \mathrm{Cl}$ represented by vertical bars, with 'never achieved' and 'never active' as reference categories, adjusted for skin colour, family income at birth, maternal schooling, birth weight, maternal smoking during the pregnancy, maternal age at birth, breast-feeding duration and current height

BMD in comparison to those observed for lumbar-spine BMD in the relationship with daily frequency of consumption of dairy products, whereas PA coefficients were greater in magnitude in relation to lumbar-spine BMD. Consumption of dairy products and PA at 4 years of age were positively related to BMD in both anatomical sites, with similar results at 6 years. Maintenance of adequate consumption of dairy products and high PA in a 2 -year period was beneficial to BMD at both sites in boys. Results seemed to be more consistent among boys than girls although statistical tests for interaction were significant only for consumption of dairy products, possibly indicating a sex difference already from childhood that may manifest in substantial sex differences by age.

The Dietary Reference Intakes established by the Institute of Medicine ${ }^{(16)}$ recommend a daily Ca intake of $800 \mathrm{mg} / \mathrm{d}$ for children aged 4-8 years. This recommendation is exemplified as a daily consumption of $480 \mathrm{ml}$ of milk or two or three portions of dairy products ${ }^{(9,17)}$. The premise of this recommendation is that milk during childhood has a positive impact on current and future bone health ${ }^{(18)}$. Maintenance of adequate $\mathrm{Ca}$ intake at young ages positively predicted BMD status in prepubertal and postpubertal years $^{(19)}$. Daily consumption of two or more servings of dairy products starting at 3-5 years of age was positively associated with higher BMD and bone area in adolescents aged $15-17$ years $^{(20)}$. Besides, the positive effect of dairy consumption on bone mass accretion or density in childhood is also supported by 1- to 2-year randomized trials supplementing dairy foods or milk extract in prepubertal children with low baseline $\mathrm{Ca}$ intake ${ }^{(21,22)}$.

We observed a decrease in the frequency of milk consumption followed by an increase in yoghurt and cheese consumption from 4 to 6 years old (Table 1 ). This finding corroborates a previous study that reported a positive time trend in cheese and yoghurt consumption compensated for the decrease in milk during childhood ${ }^{(23)}$. Although the consumption of dairy products seems more beneficial for bone mass accretion than Ca supplements ${ }^{(3)}$, children who decrease daily milk consumption less frequently meet the total $\mathrm{Ca}$ recommendation, which may indicate replacement by smaller dairy portion sizes or milk-based beverages with lower Ca content ${ }^{(19)}$.

Our study provided evidence of a positive association between consumption of dairy products and total-body and lumbar-spine BMD at early age. Daily consumption of dairy products during childhood is also reported as a positive dietary factor associated with greater BMD in adulthood ${ }^{(24)}$, but evidence is still inconsistent ${ }^{(25,26)}$. When children's diet is supplied with Ca through dietary products, studies report a gain in total-body BMD, especially at the lumbar spine and hip ${ }^{(21,27,28)}$. By contrast, long-term deprivation of milk in childhood is suggested as a risk factor for smaller skeleton size and significantly lower bone area, total-body and sitespecific $\mathrm{BMD}^{(29,30)}$, although lower bone area may be a consequence of small body size. 
Several studies have shown the beneficial effect of PA in pre- and postpubertal childhood on bone mass ${ }^{(31-34)}$. Results from prospective studies in young children are scarce but cross-sectional analyses suggest positive associations between PA and BMD, suggesting a short-term benefit ${ }^{(35,36)}$. Our observations extend these observations, including a 2-year follow-up period.

Although PA in young adulthood is positively related to an increase in adult $\mathrm{BMD}^{(37,38)}$, some authors describe the growth period (childhood and adolescence) as the best opportunity to improve bone mass ${ }^{(39-42)}$. However, there is no consensus on whether the effect of PA is most prominent before or after puberty although some have suggested that PA during the most active period of maturity plays an important role in optimizing bone mass ${ }^{(41,42)}$. On the other hand, it has also been suggested that the prepubertal years, in which the presence of growth hormone is more expressive than of sex steroids, is a sensitive period to increase $\mathrm{BMD}^{(43)}$. Future follow-up of the current cohort throughout puberty may resolve these issues.

PA is especially important to promote increases in BMD due to mechanical loading. Different mechanisms are related to increase in BMD during puberty in boys and girls, whereas an increase in periosteal apposition in both sexes is suggested to be the main mechanism related to increase in BMD due to mechanical loading prior to puberty ${ }^{(44)}$. This may explain the lack of statistical interaction between PA and sex in association with BMD in the current cohort. The magnitude of associations between PA and BMD was greater in boys than in girls based on maternal proxy report. However, even if boys and girls are classified in the same category by maternal proxy report, the higher overall PA $(64.0 v .54 .8 \mathrm{mg})$ and time spent in MVPA ( $55.6 v .33 .3 \mathrm{~min}$ ) assessed by accelerometry at age 6.8 years observed in boys indicate differences in objectively measured PA for the same maternal perception of PA. This may potentially contribute to the difference in the magnitude of association between sexes.

Previous randomized controlled trials and studies in adults have shown that the magnitude of the association between PA and BMD is greater for weight-bearing sites, such as the lumbar spine and femoral neck ${ }^{(5,38,45)}$. These sites are more susceptible to bone adaptation promoted by loading induced by weight-bearing $\mathrm{PA}^{(45)}$. Our findings are in line with the literature since greater magnitudes of association for both longitudinal and cross-sectional analyses between maternally reported PA and objectively measured PA with BMD were observed for the lumbar spine site than for total-body BMD. In contrast, the magnitude of association was similar between the two anatomical sites for consumption of dairy products, suggesting a similar association between accrual and growth of the bone at both anatomical sites and the consumption of dairy products.

Our results on an association between consumption of dairy products and PA and BMD in children from Latin
America are novel. This is because BMD is influenced by ethnicity $^{(46,47)}$ and possibly also by socio-economic status ${ }^{(48,49)}$, factors that may differ substantially between lowor middle-income countries and high-income countries, in which previous studies have been conducted. Further, types and amounts of dairy products consumed and PA levels may also differ between low- or middle-income countries and high-income countries, as previously observed $^{(50)}$.

Some limitations should be acknowledged when interpreting our observations. First is the use of different methods to estimate the consumption of dairy products at 4 and 6 years of age. The use of only one $24 \mathrm{~h}$ dietary recall is a common practice in population-based studies, although the use of multiple $24 \mathrm{~h}$ dietary recalls increases the accuracy of the method ${ }^{(51)}$. However, a previous study examined the first three components defined by principal component analysis from a $24 \mathrm{~h}$ food recall for children and found that the results were very similar to those obtained using the $\mathrm{FFQ}^{(52)}$. In addition, as a monotonous diet was previously reported in Brazilian children ${ }^{(53,54)}$, the use of one single previous $24 \mathrm{~h}$ food recall likely reflects the food habits of the children. Other limitations are the absence of information on BMD at age 4 years, limiting longitudinal inferences. Not all children provided valid data on objectively measured PA, which may influence the results. However, our study is still one of the largest to date combining objectively measured PA with data on BMD from DXA scans in young children. Finally, we cannot exclude the possibility that our results are explained by unmeasured (e.g. genotype) or poorly measured confounders.

The use of maternal perception of her child's PA may also influence our results. Measuring self-reported PA in children is a challenge and the use of accelerometers is preferable. However, due to logistical and cost reasons, objective measures were available only at 6 years of age. On the other hand, self-reported PA was positively associated with accelerometry in a previous study carried out with children from the same city, with correlation coefficients similar to those found for other self-reported instruments used at different ages ${ }^{(10)}$, although the use of this subjective method is an important limitation. Furthermore, an increase in acceleration and time spent in MVPA was positively related to mothers' perception of PA, mainly in boys (data not shown). This reduces the risk of bias due to self-reported PA, although accelerometry does not provide information on the kind of PA performed.

Results from accelerometers showed two interesting findings: overall amount of PA was favourably related to BMD; however, bouts of at least moderate-intensity may also contribute to enhanced BMD, whereas accumulating MVPA in shorter epochs appears unrelated to BMD. Further studies are warranted to confirm these observations.

As previously described in the 'Methods', body weight was not included in the adjusted analysis since it was not 
related to total-body $\mathrm{BMD}(P=0.178$ in boys; $P=0.610 \mathrm{in}$ girls) or lumbar-spine BMD $(P=0.397$ in boys; $P=0.700$ in girls). In addition, even in the case of an association, since body weight is influenced by energy expenditure from PA and by food intake, body weight could be a mediating variable in the analyses. Thus, body weight did not meet the criteria to be considered a possible confounder in our analyses.

\section{Conclusion}

In conclusion, consumption of dairy products was positively associated with BMD in the total body and at the lumbar spine in young Brazilian children. PA assessed by maternal proxy report (only in boys) and objectively measured by accelerometry (overall PA and time spent in MVPA using $10 \mathrm{~min}$ bouts) was also positively associated with BMD, particularly at the lumbar spine site. These findings support the evidence of a cumulative effect of PA and consumption of dairy products on bone mineral accrual during growth.

\section{Acknowledgements}

Acknowledgements: This article is based on data from the study 'Pelotas Birth Cohort, 2004' conducted by the Postgraduate Program in Epidemiology at Universidade Federal de Pelotas, with the collaboration of the Brazilian Public Health Association (ABRASCO). Financial support: The 2004 birth cohort study was supported from 2009 to 2013 by the Wellcome Trust through the programme entitled 'Major Awards for Latin America on Health Consequences of Population Change' (grant number 086974/Z/08/Z). The WHO (grant number $03014 \mathrm{HNI}$ ), the National Support Program for Centers of Excellence (PRONEX) (grant number 04/0882.7), the Brazilian National Research Council (CNPq) (grant numbers 481012-2009-5, 484077-2010-4, 470965-2010-0 and 481141-2007-3), the Brazilian Ministry of Health (grant number 25000.105293/2004-83) and Children's Pastorate have supported previous phases of the study. The funders had no role in the design, analysis or writing of this article. Conflict of interest: The authors declare no conflict of interests according to International Committee of Medical Journal Editors' guidelines. Authorship: R.M.B. conceptualized the study and conducted the statistical analysis. R.M.B., J.S.V., M.R.D., A.M., I.S.S., U.E. and B.L.H. wrote the manuscript. A.M., I.S.S. and B.L.H. coordinated the last follow-up with participants. U.E. supervised the accelerometry analysis. All authors approved the final version. Ethics of human subject participation: This study was conducted according to the guidelines laid down in the Declaration of Helsinki and all follow-ups of the Pelotas 2004 Birth Cohort Study were approved by the Ethics Committee of the Federal University of Pelotas
Medical School. All mothers signed an informed consent before any data collection.

\section{Supplementary material}

To view supplementary material for this article, please visit https://doi.org/10.1017/S1368980018001258

\section{References}

1. Kuh D; New Dynamics of Ageing Preparatory Network (2007) A life course approach to healthy aging, frailty, and capability. J Gerontol A Biol Sci Med Sci 62, 717-721.

2. Heaney RP, Abrams S, Dawson-Hughes B et al. (2000) Peak bone mass. Osteoporosis Int 11, 985-1009.

3. Cheng S, Lyytikainen A, Kroger H et al. (2005) Effects of calcium, dairy product, and vitamin D supplementation on bone mass accrual and body composition in 10-12-y-old girls: a 2-y randomized trial. Am J Clin Nutr 82, 1115-1126.

4. Rizzoli R (2014) Dairy products, yogurts, and bone health. Am J Clin Nutr 99, Suppl. 5, 1256S-1262S.

5. French SA, Fulkerson JA \& Story M (2000) Increasing weight-bearing physical activity and calcium intake for bone mass growth in children and adolescents: a review of intervention trials. Prev Med 31, 722-731.

6. Meyer U, Romann M, Zahner L et al. (2011) Effect of a general school-based physical activity intervention on bone mineral content and density: a cluster-randomized controlled trial. Bone 48, 792-797.

7. Gunter KB, Almstedt HC \& Janz KF (2012) Physical activity in childhood may be the key to optimizing lifespan skeletal health. Exerc Sport Sci Rev 40, 13-21.

8. Santos IS, Barros AJ, Matijasevich A et al. (2014) Cohort profile update: 2004 Pelotas (Brazil) Birth Cohort Study. Body composition, mental health and genetic assessment at the 6 years follow-up. Int J Epidemiol 43, 1437-1437a-f.

9. Ministério da Saúde (2005) Guia Alimentar para a População Brasileira. Brasília: Coordenação-Geral da Política de Alimentação e Nutrição, Secretaria de Atenção à Saúde, Ministério da Saúde.

10. Bielemann RM, Reichert FF, Paniz VM et al. (2011) Validation of the Netherlands physical activity questionnaire in Brazilian children. Int J Behav Nutr Phys Act 8, 45.

11. Knuth AG, Assuncao MC, Goncalves H et al. (2013) Methodological description of accelerometry for measuring physical activity in the 1993 and 2004 Pelotas (Brazil) birth cohorts. Cad Saude Publica 29, 557-565.

12. da Silva IC, van Hees VT, Ramires VV et al. (2014) Physical activity levels in three Brazilian birth cohorts as assessed with raw triaxial wrist accelerometry. Int J Epidemiol $\mathbf{4 3}$, 1959-1968.

13. Sabia S, van Hees VT, Shipley MJ et al. (2014) Association between questionnaire- and accelerometer-assessed physical activity: the role of sociodemographic factors. $A m J$ Epidemiol 179, 781-790.

14. van Hees VT, Gorzelniak L, Dean Leon EC et al. (2013) Separating movement and gravity components in an acceleration signal and implications for the assessment of human daily physical activity. PLoS One 8, e61691.

15. Hildebrand M, van Hees VT, Hansen BH et al. (2014) Age group comparability of raw accelerometer output from wrist- and hip-worn monitors. Med Sci Sports Exerc 46, $1816-1824$.

16. Institute of Medicine, Food and Nutrition Board (1998) Dietary Reference Intakes: A Risk Assessment Model for 
Establishing Upper Intake Levels for Nutrients. Washington, DC: National Academy of Sciences.

17. US Department of Agriculture \& US Department of Health and Human Services (2010) Dietary Guidelines for Americans, 7th ed. Washington, DC: US Government Printing Office.

18. Kalkwarf HJ, Zemel BS, Gilsanz V et al. (2007) The bone mineral density in childhood study: bone mineral content and density according to age, sex, and race. J Clin Endocrinol Metab 92, 2087-2099.

19. Fisher JO, Mitchell DC, Smiciklas-Wright H et al. (2004) Meeting calcium recommendations during middle childhood reflects mother-daughter beverage choices and predicts bone mineral status. Am J Clin Nutr 79, 698-706.

20. Moore LL, Bradlee ML, Gao D et al. (2008) Effects of average childhood dairy intake on adolescent bone health. J Pediatr 153, 667-673.

21. Bonjour JP, Carrie AL, Ferrari S et al. (1997) Calciumenriched foods and bone mass growth in prepubertal girls: a randomized, double-blind, placebo-controlled trial. J Clin Invest 99, 1287-1294.

22. Huncharek M, Muscat J \& Kupelnick B (2008) Impact of dairy products and dietary calcium on bone-mineral content in children: results of a meta-analysis. Bone $\mathbf{4 3}, 312-321$.

23. Dror DK \& Allen LH (2014) Dairy product intake in children and adolescents in developed countries: trends, nutritional contribution, and a review of association with health outcomes. Nutr Rev 72, 68-81.

24. Wadolowska L, Sobas K, Szczepanska JW et al. (2013) Dairy products, dietary calcium and bone health: possibility of prevention of osteoporosis in women: the Polish experience. Nutrients 5, 2684-2707.

25. Feskanich D, Willett WC \& Colditz GA (2003) Calcium, vitamin D, milk consumption, and hip fractures: a prospective study among postmenopausal women. Am J Clin Nutr 77, 504-511.

26. Matsuzaki M, Kuper H, Kulkarni B et al. (2014) Life-course determinants of bone mass in young adults from a transitional rural community in India: the Andhra Pradesh Children and Parents Study (APCAPS). Am J Clin Nutr 99, 1450-1459.

27. Matkovic V, Landoll JD, Badenhop-Stevens NE et al. (2004) Nutrition influences skeletal development from childhood to adulthood: a study of hip, spine, and forearm in adolescent females. J Nutr 134, issue 3, 701S-705S.

28. Specker B \& Binkley T (2003) Randomized trial of physical activity and calcium supplementation on bone mineral content in 3- to 5-year-old children. J Bone Miner Res 18, $885-892$.

29. Black RE, Williams SM, Jones IE et al. (2002) Children who avoid drinking cow milk have low dietary calcium intakes and poor bone health. Am J Clin Nutr 76, 675-680.

30. Pettifor JM \& Moodley GP (1997) Appendicular bone mass in children with a high prevalence of low dietary calcium intakes. J Bone Miner Res 12, 1824-1832.

31. Bailey DA, McKay HA, Mirwald RL et al. (1999) A six-year longitudinal study of the relationship of physical activity to bone mineral accrual in growing children: the University of Saskatchewan bone mineral accrual study. J Bone Miner Res 14, 1672-1679.

32. Janz KF, Gilmore JM, Levy SM et al. (2007) Physical activity and femoral neck bone strength during childhood: the Iowa Bone Development Study. Bone 41, 216-222.

33. Janz KF, Letuchy EM, Francis SL et al. (2014) Objectively measured physical activity predicts hip and spine bone mineral content in children and adolescents ages $5-15$ years: Iowa Bone Development Study. Front Endocrinol 5, 112.
34. Michalopoulou M, Kambas A, Leontsini D et al. (2013) Physical activity is associated with bone geometry of premenarcheal girls in a dose-dependent manner. Metabolism 62, 1811-1818.

35. Harvey NC, Cole ZA, Crozier SR et al. (2012) Physical activity, calcium intake and childhood bone mineral: a population-based cross-sectional study. Osteoporos Int 23, $121-130$.

36. Janz KF, Burns TL, Torner JC et al. (2001) Physical activity and bone measures in young children: the Iowa Bone Development Study. Pediatrics 107, 1387-1393.

37. Bielemann RM, Domingues MR, Horta BL et al. (2014) Physical activity from adolescence to young adulthood and bone mineral density in young adults from the 1982 Pelotas (Brazil) Birth Cohort. Prev Med 62, 201-207.

38. Bielemann RM, Martinez-Mesa J \& Gigante DP (2013) Physical activity during life course and bone mass: a systematic review of methods and findings from cohort studies with young adults. BMC Musculoskelet Disord 14, 77.

39. Boreham CA \& McKay HA (2011) Physical activity in childhood and bone health. Br J Sports Med 45, 877-579.

40. Karlsson MK (2007) Does exercise during growth prevent fractures in later life? Med Sport Sci 51, 121-136.

41. Khan K, McKay HA, Haapasalo H et al. (2000) Does childhood and adolescence provide a unique opportunity for exercise to strengthen the skeleton? J Sci Med Sport 3, 150-164.

42. Ondrak KS \& Morgan DW (2007) Physical activity, calcium intake and bone health in children and adolescents. Sports Med 37, 587-600.

43. Bass SL (2000) The prepubertal years: a uniquely opportune stage of growth when the skeleton is most responsive to exercise? Sports Med 30, 73-78.

44. Kriemler S, Zahner L, Puder JJ et al. (2008) Weight-bearing bones are more sensitive to physical exercise in boys than in girls during pre- and early puberty: a cross-sectional study. Osteoporos Int 19, 1749-1758.

45. Guadalupe-Grau A, Fuentes T, Guerra B et al. (2009) Exercise and bone mass in adults. Sports Med 39, 439-468.

46. Freedman BI \& Register TC (2012) Effect of race and genetics on vitamin D metabolism, bone and vascular health. Nat Rev Nephrol 8, 459-466.

47. Leslie WD (2012) Clinical review: ethnic differences in bone mass - clinical implications. J Clin Endocrinol Metab 97, 4329-4340.

48. Brennan SL, Henry MJ, Wluka AE et al. (2009) BMD in population-based adult women is associated with socioeconomic status. J Bone Miner Res 24, 809-815.

49. Brennan SL, Pasco JA, Urquhart DM et al. (2011) Association between socioeconomic status and bone mineral density in adults: a systematic review. Osteoporos Int 22, 517-527.

50. Hallal PC, Andersen LB, Bull FC et al. (2012) Global physical activity levels: surveillance progress, pitfalls, and prospects. Lancet 380, 247-57.

51. Tucker KL (2007) Assessment of usual dietary intake in population studies of gene-diet interaction. Nutr Metab Cardiovas Dis 17, 74-81.

52. Robinson S, Marriott L, Poole J et al. (2007) Dietary patterns in infancy: the importance of maternal and family influences on feeding practice. Br J Nutr 98, 1029-1037.

53. Farias Júnior G \& Osório MM (2005) Alimentary profile of under-five year old children. Rev Nutr 18, 10.

54. Santos I, Victora CG, Martines J et al. (2001) Nutrition counseling increases weight gain among Brazilian children. J Nutr 131, 2866-2873. 